Artículo original

\title{
¿Qué hemos aprendido sobre la muñeca en los últimos años? Del laboratorio a la práctica clínica
}

\author{
What have we learned about the wrist in recent years? From lab \\ to clinical practice
}

\author{
Àlex Lluch", Mireia Esplugas", Ana Carreño', Guillem Salvà2, Inma Puig de la Bellacasa ${ }^{3}$, Núria \\ Fernández ${ }^{4}$, Manuel Llusá ${ }^{\text {, Alfonso Rodríguez-Baeza }}{ }^{6,}$ Elisabet Hagert’, Marc Garcia-Elias ${ }^{1}$ \\ ${ }^{1}$ Institut Kaplan - Barcelona - España. \\ ${ }^{2}$ Hospital Son Espases - Palma de Mallorca - España. \\ ${ }^{3}$ Hospital Mútua de Terrassa - Terrassa - España. \\ ${ }^{4}$ Hospital Universitario Josep Trueta - Girona - España. \\ ${ }^{5}$ Universitat de Barcelona - Departament d'Anatomia i Embriologia Humana - Barcelona - España. \\ ${ }^{6}$ Universitat Autònoma de Barcelona - Departament de Ciències Morfològiques - Barcelona - España. \\ 7 Arcademy, Sophiahemmet Hospital - Karolinska Institutet - Estocolmo - Suecia. \\ Correspondencia: Àlex Lluch. Hospital Universitario Vall d'Hebron. Teléfono: +34 620214202. Barcelona. España. \\ Correo electrónico: alluch@vhebron.net.
}

\section{Información del artículo Resumen}

Recibido: 23/12/2020

Aceptado: 14/04/2021

Palabras clave: Muñeca; Biomecánica; Carga; Cinética; Cinemática; Control neuromuscular; Inestabilidad.

Keywords: Wrist; Biomechanics; Load; Kinetics; Kinematics; Neuromuscular control; Instability.

DOI: https://doi.org/10.25214/28056272.1181
Hace más de una década, un grupo de amigos interesados en la anatomía y la biomecánica de la muñeca empezamos a pasar horas en los departamentos de Anatomía de las dos universidades más importantes de Barcelona sin esperar otra recompensa que aprender y saciar nuestras inquietudes. Aprovechando el lanzamiento de la Revista de Cirugía de Mano y Microcirugía de la Asociación Colombiana de Cirugía de la Mano, hemos decidido hacer un balance del aprendizaje acumulado. Independientemente de los conocimientos que hemos adquirido y difundido, la principal conclusión de este balance es la necesidad del trabajo en equipo para avanzar. Como todo equipo, el nuestro también necesitaba de capitanes que lo liderasen, y tuvimos la suerte y el honor de contar con el doctor Marc Garcia-Elias como guía y los profesores Manuel Llusá y Alfonso Rodríguez Baeza como apoyo incondicional. Este escrito es un pequeño resumen del trabajo de nuestro grupo durante estos más de 11 años en el Laboratorio de Biomecánica de la Muñeca y sus potenciales aplicaciones en la práctica clínica.

\footnotetext{
Abstract

More than a decade ago, a group of friends interested in the Anatomy and Biomechanics of the wrist began to spend hours in the Anatomy Departments of the two most important Universities in Barcelona, without expecting any other reward than learning and satisfying our concerns. Taking advantage of the launch of the journal of Hand Surgery and microsurgery from Colombian Association of Hand Surgery, we have decided to take stock of the accumulated learning. Regardless of the knowledge that we have acquired and disseminated; the main conclusion of this balance is the need for teamwork to move forward. Like any team, ours also needed captains to lead it. We were lucky and honored to have Dr. Marc Garcia-Elias as our guide, and Professors Manuel Llusá and Alfonso Rodríguez Baeza as unconditional support. This writing is a small summary of the work of our group during these more than 11 years in the Laboratory of Biomechanics of the Wrist, and of its potential applications in the clinic.
} 


\section{Introducción: condrocalcinosis en la muñeca}

La enfermedad por depósito de cristales de pirofosfato cálcico (condrocalcinosis o pseudogota) es una causa de artropatía muy frecuente en la muñeca. Inicialmente, los cristales aparecen en el líquido sinovial; luego, se depositan en la membrana sinovial, los ligamentos y las estructuras fibrocartilaginosas, y, finalmente, se ubican en el cartílago articular ${ }^{1}$. La calcificación del fibrocartílago triangular es la imagen que clásicamente se ha considerado más específica; sin embargo, la afectación aislada de la articulación escafoidestrapecio-trapezoide (STT) y las afectaciones escafolunar (EL), radiocúbito-carpiana (RCC), lunopiramidal (LP), mediocarpiana (MC), radiolunar (RL) o pisopiramidal (PP) son otras posibles formas de presentación. Se ha descrito incluso un patrón de deterioro articular secundario a la condrocalcinosis: el patrón $\mathrm{SCAC}^{2}$, en una línea similar a los patrones SLAC y SNAC.

Los hallazgos compatibles con una afectación articular y periarticular por condrocalcinosis en las muñecas de los especímenes cadavéricos que los autores de este estudio han utilizado en sus estudios biomecánicos no han sido una excepción, sino la norma. Los cambios patológicos aumentan con la edad y son relativamente poco sintomáticos en la mayoría de casos. Eso podría explicar el gran número de muñecas afectadas pero no tratadas que se han encontrado (Figuras 1 y 2 ).

Figura 1. Calcificación del complejo fibrocartílago triangular como característica de la condrocalcinosis.

Fuente: imagen obtenida durante la realización del estudio.

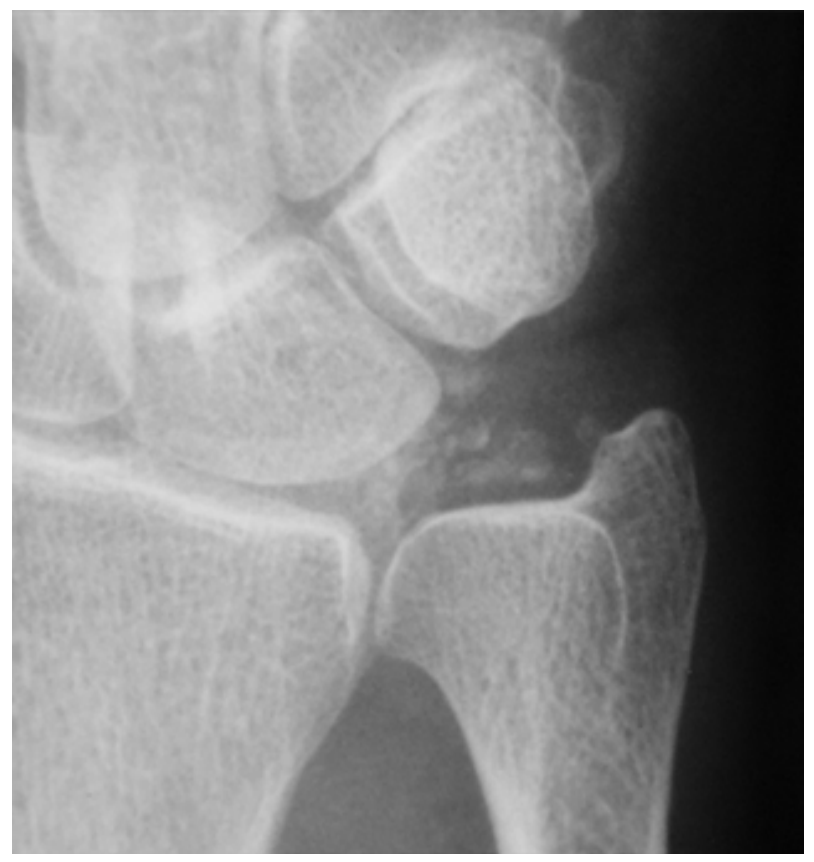

Desde un punto de vista práctico, ser conscientes de la elevada incidencia de la condrocalcinosis ha permitido tenerla en un lugar preferente en el diagnóstico diferencial de los problemas dolorosos de la muñeca no traumática, teniendo en cuenta, además, que la forma de presentación clínica es variable ${ }^{1}$.
Figura 2. Patrón SCAC en un espécimen cadavérico. Afectación radioescafoidea y mediocarpiana similar al patrón SNAC y SLAC. El hundimiento del escafoides en el radio (scalloping) es casi patognomónico.

Fuente: imagen obtenida durante la realización del estudio.

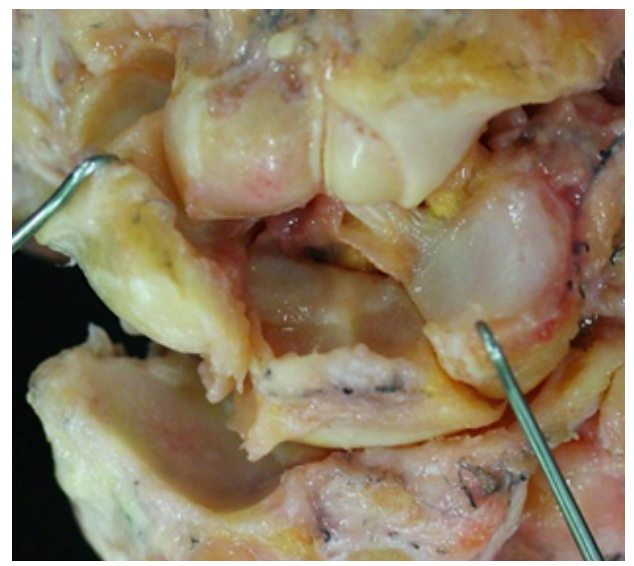

Desde un punto de vista práctico, ser conscientes de la elevada incidencia de la condrocalcinosis ha permitido tenerla en un lugar preferente en el diagnóstico diferencial de los problemas dolorosos de la muñeca no traumática, teniendo en cuenta, además, que la forma de presentación clínica es variable ${ }^{1}$.

El hallazgo de tantas muñecas con patrones degenerativos también permitió plantear la duda de si el comportamiento cinético del carpo con una artropatía secundaria a una lesión del ligamento EL es el mismo que cuando la lesión EL masiva se crea de forma artificial en el laboratorio. Aunque el comportamiento del escafoides es similar en ambos casos, la magnitud de su desplazamiento es mayor en las muñecas con una lesión crónica y cambios degenerativos. Sin embargo, el músculo extensor carpi ulnaris (ECU) tiene un papel desestabilizante de relevancia en las muñecas degenerativas, igual que ocurre en las lesiones recién creadas en el laboratorio - como se explicará en el apartado de control neuromuscular en la inestabilidad EL-, por lo que su contracción isométrica también debe evitarse en lesiones crónicas con artropatía secundaria ${ }^{3}$.

\section{Estudios sobre la cinemática carpiana}

La cinemática es la parte de la física que se ocupa del estudio de los movimientos articulares, independientemente de las fuerzas que los han generado. Una pregunta que los autores de este artículo se han planteado es cómo consigue el carpo tener tanta movilidad sin que se produzcan lesiones.

El movimiento denominado del lanzador de dardos (MLD) o dart-throwing motion, cuando la muñeca se mueve de extensión e inclinación radial hacia flexión e inclinación cubital, se hace siguiendo uno de los ejes de movimiento de la muñeca más utilizados en las actividades cotidianas. Clásicamente, se ha considerado que la mayoría del MLD de una muñeca sana se da a nivel de la articulación mediocarpiana y que, durante este, la hilera proximal del carpo se mantiene prácticamente inmóvil $1^{4,5}$.

Para determinar si el patrón de movimiento descrito se mantiene en las muñecas con una lesión completa del ligamento EL, se diseñó 
un estudio cinemático carpiano in vivo utilizando la tecnología TC 4D (proyección secuencial en el tiempo de imágenes TC 3D). Con este fin, se comparó la movilidad carpiana activa siguiendo el eje del MLD entre muñecas sanas y muñecas con una lesión EL. Los resultados del estudio demostraron que en las muñecas sanas existe una rotación de la hilera proximal del carpo no despreciable en el plano frontal, mientras que las muñecas con inestabilidad EL tienen un comportamiento diferente. $\mathrm{Si}$ los ligamentos EL son incompetentes, el escafoides aumenta de forma significativa su movilidad durante el MLD, mientras que el semilunar permanece casi inmóvil hasta el final del movimiento ${ }^{6}$ (Figura 3 ).

Figura 3. Posición extrema del movimiento del lanzador de dardos con la muñeca en flexión e inclinación cubital. El polo proximal del escafoides se desplaza hacia la zona dorsal de la fosa escafoidea, mientras el semilunar permanece poco móvil en la fosa lunar.

Fuente: imagen obtenida durante la realización del estudio.

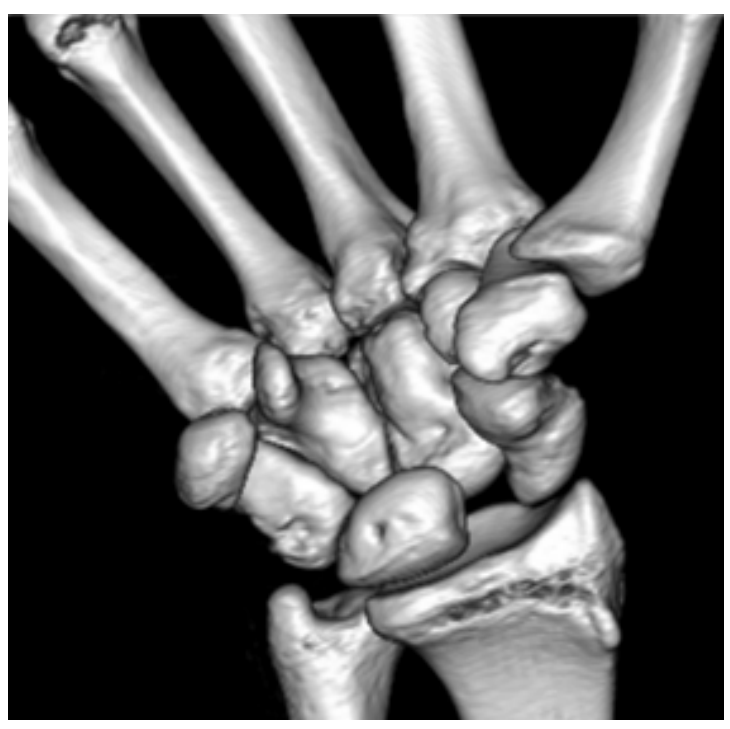

La aplicación práctica de este hallazgo es matizar la idea generalizada de que el estrés a nivel del ligamento EL no existe durante el MLD. Así pues, en el posoperatorio de las muñecas con una lesión EL reparada o en un tratamiento conservador de la disfunción EL, deben evitarse ambos extremos del MLD para no poner en tensión el complejo EL.

\section{Estudios sobre cinética carpiana}

La cinética o dinámica es la rama de la física que se ocupa de los efectos de las fuerzas sobre los cuerpos materiales. A continuación, se revisan algunos estudios relacionados con dos tipos de fuerza aplicados sobre el carpo: las fuerzas de compresión axial y las fuerzas de tracción axial.

\section{Efecto cinético de la carga axial del carpo}

En el laboratorio, una muñeca recibe una carga axial cuando se le aplica una fuerza externa de distal a proximal a lo largo del metacarpiano del dedo medio con la muñeca en posición neutra.

La forma clásica de comprender cómo los huesos de la hilera proximal del carpo son capaces de soportar una carga axial sin colapsar es imaginar que son un muelle. El escafoides sería uno de los brazos del muelle, el piramidal el otro y el semilunar se mantendría en el centro. Cuando el muelle se somete a una carga axial, el desplazamiento hacia un sentido de uno de los brazos (flexión del escafoides) se compensa con el desplazamiento en sentido contrario del otro brazo (extensión del piramidal), lo que estabiliza la zona central del muelle (semilunar) (Figuras 4 y 5).

Figuras 4 y 5. Flexión del escafoides y extensión del piramidal bajo carga axial que confieren la estabilidad de la hilera proximal.

Fuente: elaboración propia.

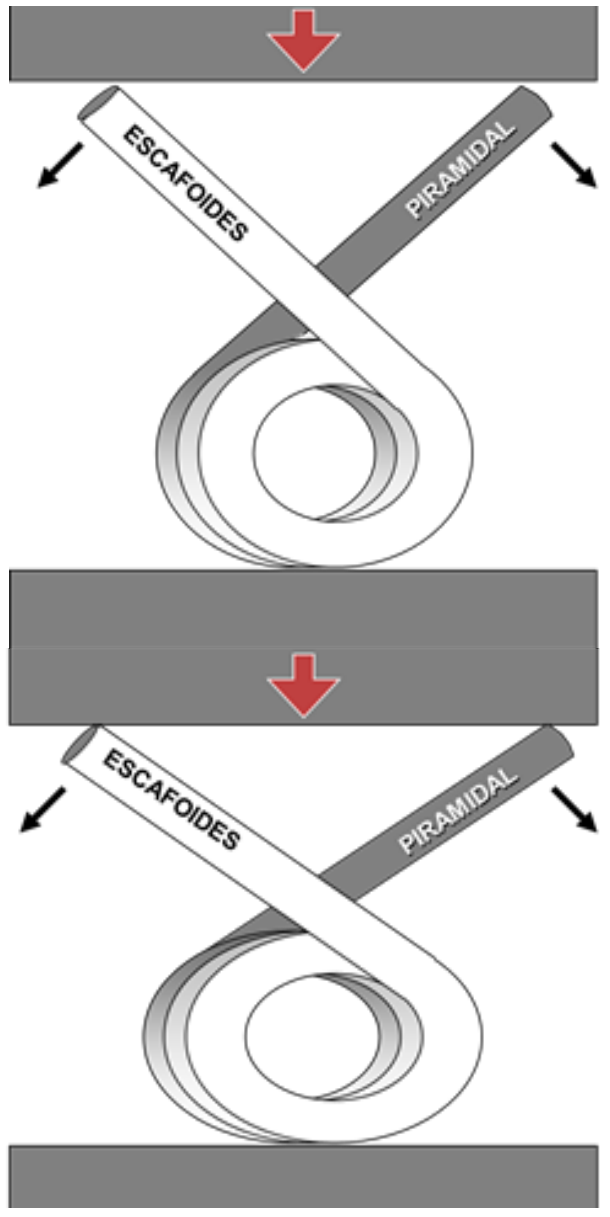

Para confirmar el comportamiento de los huesos del carpo bajo carga axial se utilizó un soporte especialmente diseñado que mantiene la muñeca y el carpo en posición neutra en todo momento. Además, se usó el sistema Fastrak® (Polhemus Inc., Colchester, EE. UU.) para monitorizar los desplazamientos y las rotaciones angulares que pueden sufrir los huesos de las dos hileras carpianas bajo la carga axial (Figura 6). Este sistema crea un campo electromagnético con el que es posible registrar la posición y la orientación de unos marcadores específicos de movimiento (sensores) en relación con un sistema fijo de coordenadas (transmisor). El sistema ha sido validado previamente para estudios biomecánicos y es el que los autores de este artículo han utilizado para todos los análisis cinéticos que han realizado ${ }^{7}$ (Figuras 7 y 8 ). 
Figura 6. Esquema del estudio sobre carga axial. El peso se aplica sobre el tercer metacarpiano y los desplazamientos del capitate, el escafoides y el piramidal se registran con

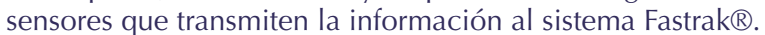
Fuente: elaboración propia.

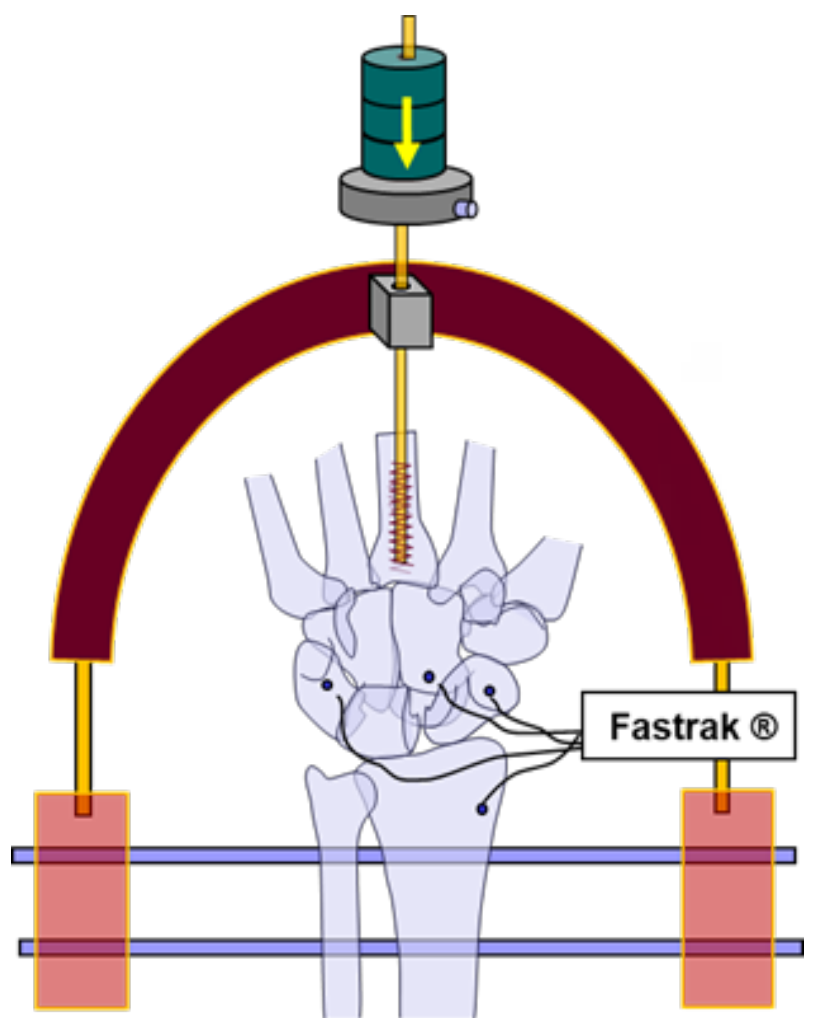

Figura 7. Detalle de los sensores. Se estabilizan en los huesos carpianos con tornillos de material plástico para que no generen interferencias con el campo magnético en el que se basa el sistema de registro.

Fuente: imagen obtenida durante la realización del estudio.

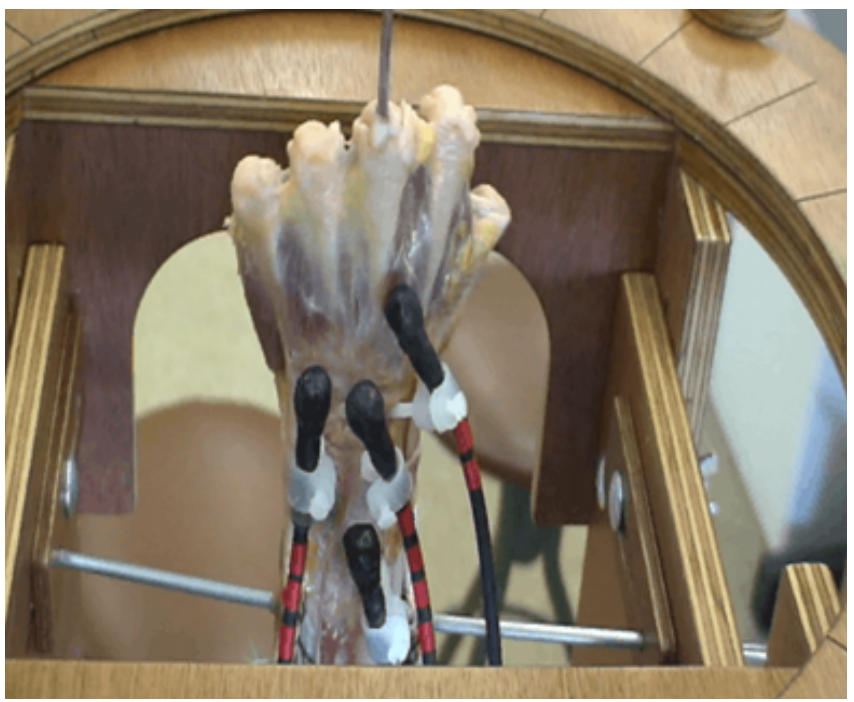

Figura 8. Sensores y emisor conectados al sistema Fastrak ${ }^{\circledR}$. El sistema informa de la posición espacial de los sensores y de los cambios en esta tras aplicar la carga.

Fuente: imagen obtenida durante la realización del estudio.

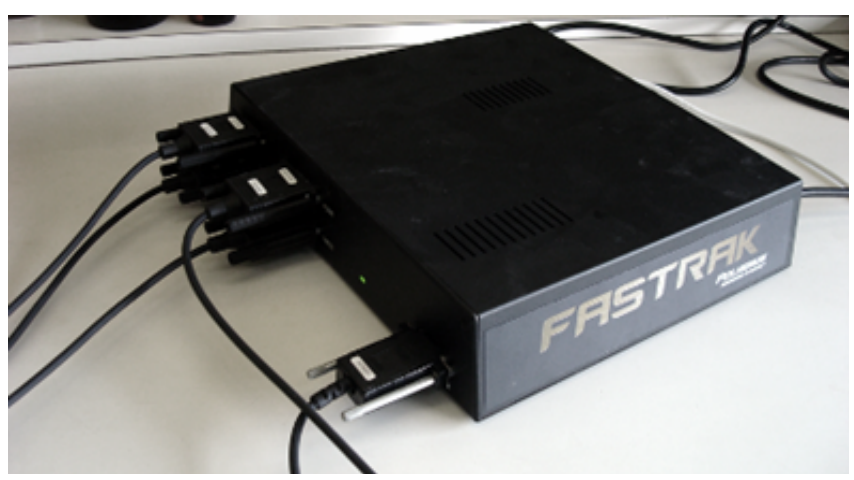

En primer lugar, los experimentos de carga axial sobre un carpo fijado en posición neutra demostraron que dicha carga genera una rotación en sentido interno (pronación) de la hilera distal del carpo a través de la articulación mediocarpiana, una flexión del escafoides, una extensión del piramidal y una traslación hacia cubital de todo el carpo. Estos resultados difieren de la concepción clásica del muelle. Bajo una carga axial, el carpo halla una posición de autobloqueo iniciada con una pronación intracarpiana, lo que le permite transmitir la fuerza de la carga axial desde distal a proximal sin colapsar.

En segundo lugar, cuando el complejo EL está intacto, el centroide de presión radioescafoideo se desplaza ligeramente hacia palmar mientras se mantiene siempre en un área de la fosa escafoidea con un buen grosor de cartílago articular. Por el contrario, cuando existe una lesión EL completa, la carga axial desplaza este mismo centroide de presión hacia el dorso de la fosa escafoidea, que es una zona con poco cartílago y mal preparada para soportar cargas.

Sumado a las alteraciones cinemáticas que aparecen en las disfunciones del complejo EL, este hallazgo ayuda a entender que los cambios artrósicos secundarios a una inestabilidad EL de larga evolución (SLAC) se inician entre el polo proximal del escafoides y la zona dorsal de la fosa escafoidea del radio.

Otra conclusión práctica de estos hallazgos es que, tras hacer una ligamentoplastia EL, lo importante no es corregir la separación (gap) entre el escafoides y el semilunar en el plano coronal, sino corregir la flexión y la pronación del escafoides (en los planos de sagital y axial, respectivamente), movimientos que hacen que el escafoides ejerza más presión en una área de superficie de cartílago menor y peor preparada para la carga.

\section{Efecto cinético de la tracción axial del carpo}

La alineación de los huesos carpianos cuando la muñeca es sometida a tracción axial no había sido estudiada previamente en el laboratorio. Para valorarla se utilizó el mismo sistema de estabilización de la muñeca que en los estudios de carga axial y el sistema Fastrack ${ }^{\circledR}$ de registro posicional con sensores insertados en los huesos de la primera y la segunda hilera del carpo. En esta ocasión, sin embargo, se aplicaron fuerzas externas de tracción axial sobre el metacarpiano del dedo medio con la muñeca fijada en posición neutra (Figura 9). 
El experimento se realizó sobre muñecas sanas y sobre muñecas con una rotura completa del complejo EL provocada.

Figura 9. Esquema del estudio sobre tracción axial. A través de un sistema de poleas se transmite la tracción que genera un peso al tercer metacarpiano.

Fuente: Elaboración propia.

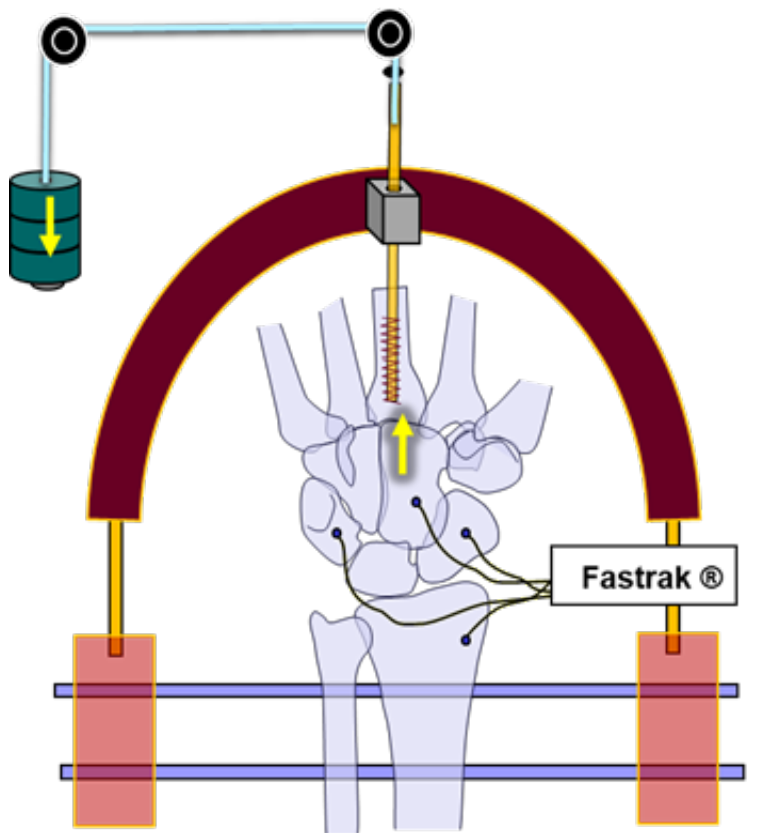

Los resultados demostraron que, incluso en las muñecas con una lesión amplia del ligamento EL, la tracción axial del carpo restablece la alineación de todas las articulaciones periescafoideas ( $\mathrm{RC}$, $\mathrm{MC}$ y EL) en los tres planos del espacio, ya que genera una rotación externa (supinación) de la hilera distal del carpo ${ }^{8}$.

Estos resultados experimentales pueden aplicarse en el campo de la artroscopia de muñeca, donde se somete esta extremidad a fuerzas de distracción. Así, la técnica quirúrgica puede dificultar el diagnóstico de la disfunción EL basada en signos indirectos intraoperatorios (diástasis, escalones, movimientos periescafoideos), a menos que se afloje completamente la tracción que se aplica.

\section{Ligamentos isodinámicos}

¿Puede el complejo ligamentoso EL, de forma aislada, soportar todas las cargas que pasan a nivel de la articulación escafolunar?, ¿es capaz de limitar la gran movilidad del escafoides, realizada en la mayoría de las ocasiones bajo carga? Teniendo en cuenta que la porción más resistente de este complejo (dorsal) solo puede soportar unos $26 \mathrm{~kg}(260 \mathrm{~N})^{9}$, las respuestas a estas preguntas son negativas.

Cuando una muñeca recibe una carga o una tracción axial, los ligamentos intrínsecos y extrínsecos se pueden tensar, relajar o permanecer inalterados. Cuando dos o más ligamentos muestran una respuesta similar, se dice que tienen un comportamiento isodinámico. Parece lógico, por lo tanto, plantear la existencia de un conjunto de ligamentos que actúan de forma conjunta (ligamentos isodinámicos) para favorecer la absorción de cargas y evitar los desplazamientos excesivos entre los huesos carpianos cuando estas aparezcan.
Tras someter a varios especímenes a carga y tracción axial, se planteó la existencia de dos complejos de ligamentos carpianos isodinámicos:

- Complejo ligamentoso helicoidal antipronación: el HAPL, por su sigla en inglés, limitaría los desplazamientos descritos bajo carga axial y estaría compuesto por los ligamentos:

- Radiosemilunar largo, que limita la traslación cubital del carpo.

- Lunopiramidal palmar y dorsal, que limitan la extensión del piramidal.

- Escafopiramidal dorsal y escafolunar dorsal, que limitan la flexión y la pronación del escafoides.

- Radiocapitate y escafocapitate, que limitan la pronación excesiva de la hilera distal.

Este complejo formaría la primera línea de defensa del carpo contra el colapso generado por una carga axial o por una hiperpronación forzada de la hilera distal con el antebrazo fijo ${ }^{10}$ (Figura 10).

Figura 10. Complejo ligamentoso helicoidal antipronación. Fuente: Elaboración propia.

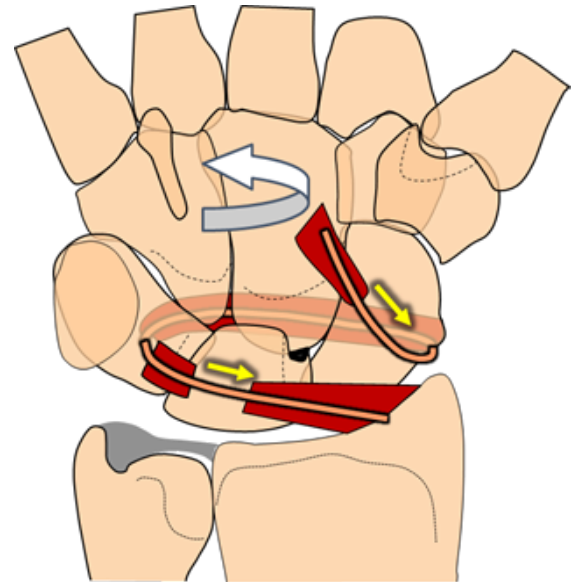

- Complejo ligamentoso helicoidal antisupinación: el HASL, por su sigla en inglés, estaría formado por un grupo de ligamentos isodinámicos de la zona radial y de la zona cubital del carpo (Figura 11). Este complejo evitaría los desplazamientos generados por una fuerza de tracción distal del carpo o una hipersupinación forzada de la hilera distal.

Figura 11. Complejo ligamentoso helicoidal antisupinación de la zona cubital y de la zona radial.

Fuente: Elaboración propia.
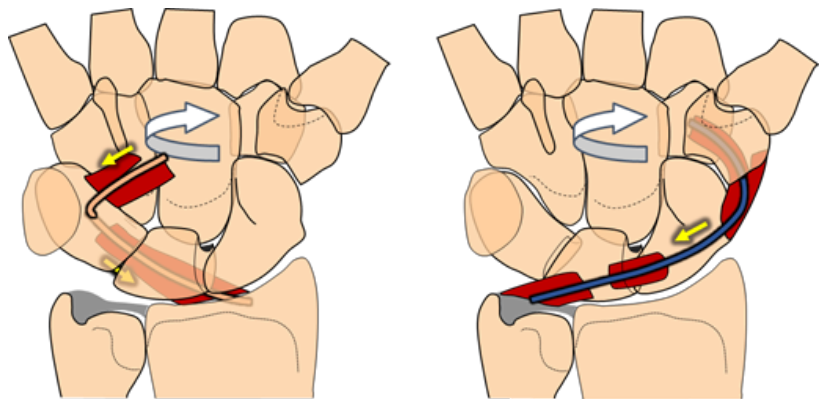
Desde un punto de vista práctico, el complejo HAPL sería importante para prevenir el colapso carpiano en pacientes con una inestabilidad EL crónica, y debería tenerse en cuenta a la hora de planificar las ligamentoplastias EL complejas (Figura 12). Por otro lado, el complejo HASL podría tener relevancia en las inestabilidades mediocarpianas ${ }^{10}$.

Figura 12. Esquema de ligamentoplastia espiral antipronación para lesiones crónicas complejas escafolunares. Se pretende recrear una espiral similar al complejo ligamentoso helicoidal antipronación.

Fuente: Elaboración propia.

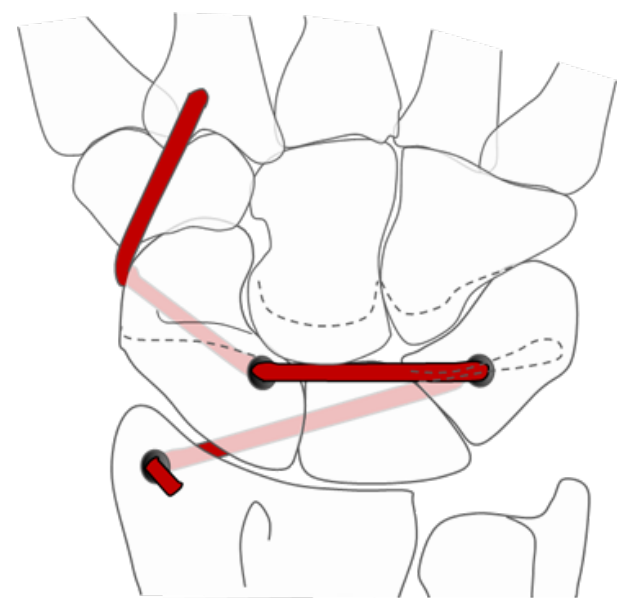

Otros estudios sobre cinética carpiana

Efecto de la carga axial tras escafoidectomía distal

Algunos casos de artropatía escafoides-trapecio-trapezoide (ETT) se presentan con una mala alineación en extensión de la hilera proximal y una subluxación dorsal compensatoria de la articulación lunocapitate (LC). En estos casos, y en otros en los que la extensión de la hilera proximal no es tan evidente, practicar una escafoidectomía distal para tratar la artropatía ETT puede empeorar de forma significativa la mala alineación.

Para dilucidar el potencial del ligamento intercarpiano dorsal (LID) en la prevención de esta complicación, se realizaron diversos experimentos de carga axial tras resecar secuencialmente el escafoides distal y el LID, y monitorizando el desplazamiento de la hilera proximal y la distal. Estos estudios concluyeron que el LID es un estabilizador secundario de la articulación $\mathrm{LC}^{11}$.

En la práctica, que el LID sea laxo o incompetente justifica la aparición de una extensión significativa del semilunar y una subluxación dorsal de la articulación mediocarpiana tras practicar una escafoidectomía distal.

Eficiencia de los tendones dadores en las ligamentoplastias EL

Para la mayoría de las ligamentoplastias EL, clásicamente se ha utilizado una bandeleta de hemitendón del músculo flexor carpi radialis (FCR). En los últimos años, los autores de este artículo han propuesto el uso del extensor carpi radialis longus (ECRL) como tendón dador, por su longitud y su potencial efecto beneficioso sobre el escafoides.
Para confirmarlo, se realizó un análisis cualitativo de ambos tendones dadores. El estudio de sus ángulos de ataque al escafoides demostró que el tendón del músculo ECRL es más eficiente que el del FCR para extender y supinar el escafoides cuando este está desconectado del semilunar ${ }^{12}$. Por tanto, se recomienda utilizar una bandeleta radial del tendón del músculo ECRL para las ligamentoplastias EL siempre que sea posible.

\section{Control neuromuscular}

Históricamente, la congruencia articular, la integridad ligamentaria y la compresión de las superficies articulares ocasionada por la contracción muscular se han considerado los tres pilares de la estabilidad del carpo. En los últimos años se ha propuesto un nuevo factor para explicar los mecanismos de estabilización carpiana, la propiocepción y el control neuromuscular.

La propiocepción es uno de los sentidos somáticos más importantes del cuerpo humano. Tradicionalmente, se han descrito tres sentidos somáticos: el dolor, el sentido termorreceptor y el sentido mecanorreceptor. La propiocepción se incluye en el tercero de los sentidos somáticos y engloba la sensación de posición y el control neuromuscular de las articulaciones.

El control neuromuscular hace referencia a la respuesta anticipatoria o inmediata de los músculos que rodean una articulación para mantener su congruencia articular. Este sentido permite que una articulación reciba cargas mucho mayores que las que sus ligamentos pueden soportar de forma aislada sin colapsar. Dicho de otra manera, si los ligamentos o los complejos de ligamentos son la primera línea de estabilización estática del carpo, la musculatura que cruza la muñeca es su segunda línea de estabilización dinámica. Estas dos líneas están conectadas a través del sistema sensoriomotor, que permite el control neuromuscular ${ }^{13,14}$.

La propiocepción en la muñeca se origina en órganos sensoriales localizados en los ligamentos y las cápsulas articulares (mecanorreceptores) ${ }^{15}$. La estimulación de estos órganos inicia un reflejo involuntario que provoca una respuesta muscular selectiva orientada a proteger la zona de la muñeca donde se ha originado la señal aferente ${ }^{16}$. En un estudio con voluntarios sanos, la estimulación directa del ligamento EL dorsal provocó una contracción inmediata de los músculos abductor pollicis longus (APL) y ECRL, y una inhibición simultánea de la contracción del músculo ECU. Toda respuesta desapareció al bloquear el nervio interóseo posterior ${ }^{17}$. Este estudio supuso que existe una conexión entre los ligamentos y la musculatura — es decir, entre la primera y la segunda línea de estabilización del carpo-y que esta conexión se hace a través de los nervios terminales que llegan a la articulación. Las aplicaciones clínicas de estos hallazgos recomiendan:

- Realizar abordajes quirúrgicos que intenten preservar la propiocepción de las estructuras capsulares, siempre que esto suponga un beneficio potencial para el paciente ${ }^{18}$ (Figura 13).

- Favorecer el uso de la artroscopia en la muñeca siempre que sea posible y esté indicada, ya que mantiene la propiocepción capsular y preserva los receptores cutáneos, que tienen un papel importante en la propiocepción consciente.

- Evitar los procedimientos en los que se eliminen los mecanorreceptores localizados en los ligamentos, como la termocoagulación, ya que la transmisión de información ligamento-muscular puede ser beneficiosa en muchas patologías. 
Figura 13. Esquema de una capsulotomía dorsal siguiendo planos ligamentosos capsulares y preservando la entrada del nervio interóseo posterior en la cápsula para que mantenga capacidad propioceptiva. A la derecha: exposición articular que se puede conseguir.

Fuente: Imágenes obtenidas durante la realización del estudio.
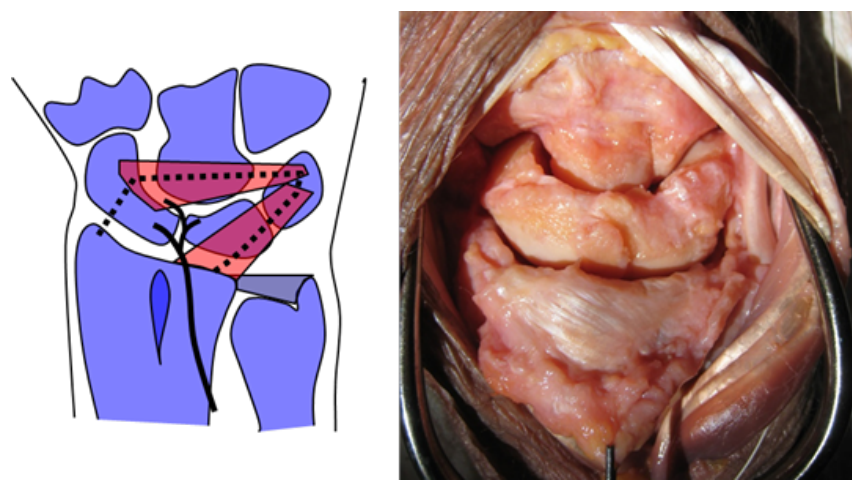

\section{Estabilización dinámica}

\section{En muñecas sanas}

Utilizando el sistema Fastrak ${ }^{\circledR}$ y simulando el efecto de los músculos motores de la muñeca mediante la carga de sus tendones distales con pesos acordes a la contracción fisiológica de sus vientres musculares, se analizó el efecto de la musculatura sobre la estabilización carpiana en muñecas sanas (Figuras 14 y 15).

Figura 14. Esquema de los estudios con carga muscular simulada. A cada tendón se anuda un peso proporcional a la fuerza de su vientre muscular para simular su contracción. Fuente: Elaboración propia.

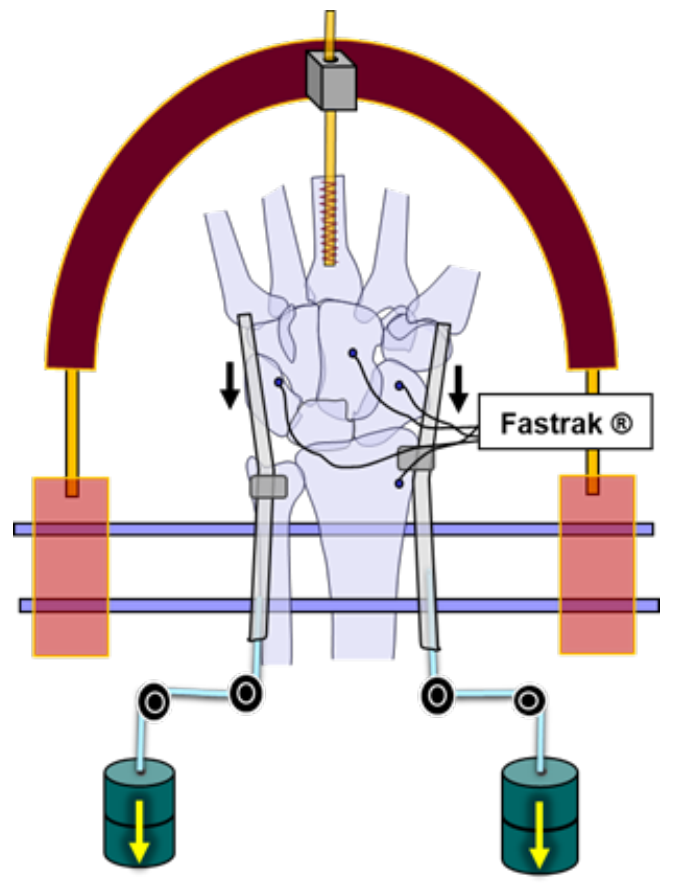

Figura 15. Pieza cadavérica fijada al soporte y preparada para un estudio de estabilización dinámica. Los pesos que traccionan los tendones están por debajo de la fotografía. Fuente: Imagen obtenida durante la realización del estudio.

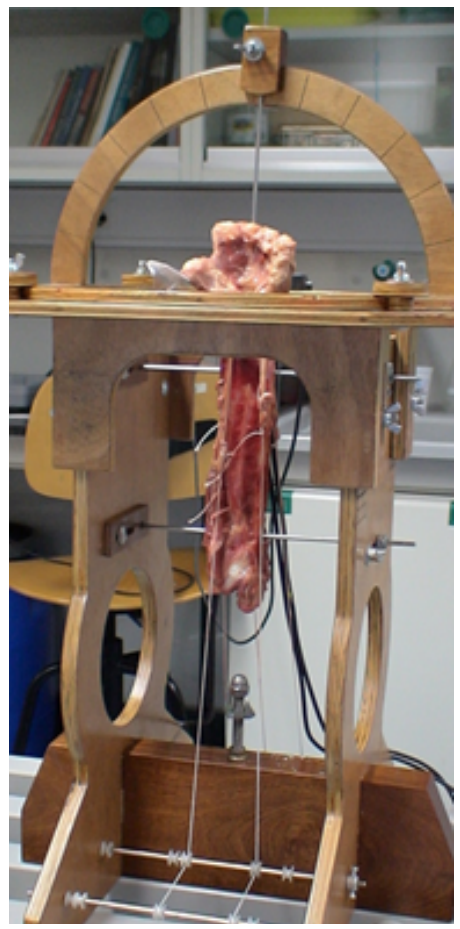

La simulación de la contracción simultánea de los cinco principales músculos motores de la muñeca (ECRL, FCR, flexor carpi ulnaris [FCU], APL y ECU) generó una supinación de la hilera distal del carpo y de la articulación mediocarpiana, y, sobre todo, una supinación y flexión de la hilera proximal ${ }^{7}$.

Además, la carga aislada del músculo ECU provocó una pronación tanto de la hilera distal como de la proximal. Por lo tanto, al ECU se le puede considerar un músculo pronador intracarpiano. Por su parte, la carga aislada de los músculos APL y ECRL indujo una supinación de la hilera distal, la articulación mediocarpiana y la hilera proximal del carpo. Por lo tanto, es posible etiquetarlos como músculos supinadores intracarpianos.

\section{Inestabilidad escafolunar}

El siguiente paso fue producir una lesión completa del complejo EL seccionando sobre el espécimen cadavérico en los ligamentos EL dorsal y volar, la membrana interósea EL, el ligamento intercarpiano dorsal y el ligamento radiosemilunar largo. Al reproducir el efecto de la carga muscular sobre el carpo se observó que:

- La carga isométrica aislada del músculo pronador intracarpiano (ECU) produjo una mala alineación de la articulación EL al inducir una pronación del escafoides en el plano sagital ${ }^{19}$ (Figura 16).

- La carga aislada o conjunta de los músculos supinadores carpianos (ECRL y APL) mejoró esta mala alineación del escafoides y generó una reducción de la articulación EL en los tres planos del espacio?.

- Estos dos efectos musculares sobre el espacio EL se mantuvieron en cualquier posición de rotación del antebrazo, aunque la 
supinación del antebrazo aumentó significativamente el efecto pronador del músculo ECU sobre la hilera distal del carpo ${ }^{20}$.

Figura 16. Efecto de la carga isométrica aislada del músculo pronador de la hilera distal por excelencia (ECU). Con la muñeca y el antebrazo fijos en posición neutra y tras haber creado una lesión EL completa, la pronación del escafoides genera una apertura franca del espacio EL (círculo). Fuente: Imagen obtenida durante la realización del estudio.

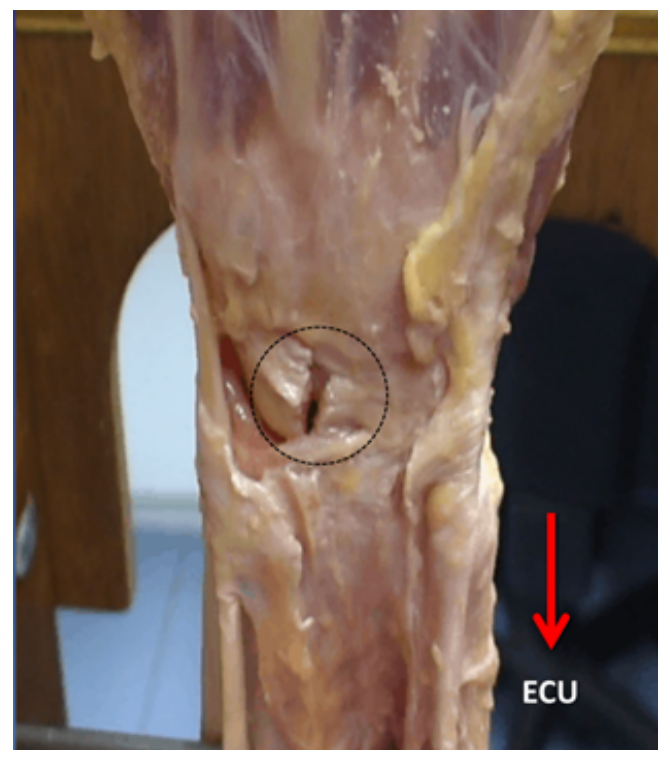

Estos hallazgos permiten concluir que el efecto beneficioso de los músculos supinadores intracarpianos debe potenciarse durante el tratamiento conservador de las disfunciones EL, en los pacientes con hiperlaxitud y sintomatología EL, y en las fases pre- y posoperatorias de las reconstrucciones EL. De hecho, ya existen protocolos y se han descrito resultados efectivos con este tipo de tratamiento ${ }^{21,22}$. El efecto desestabilizante sobre el espacio EL del músculo ECU en supinación del antebrazo puede ser utilizado para evidenciar disfunciones EL dinámicas en las que todavía no hay traducción en las radiografías estándar.

\section{Inestabilidad lunopiramidal}

Se creó una inestabilidad lunopiramidal (LP) en otro grupo de especímenes y se valoró el efecto de la carga muscular isométrica aislada o combinada sobre la articulación LP. Las conclusiones de este estudio fueron que:

- La carga isométrica de los músculos supinadores intracarpianos indujo una mala alineación del espacio LP.

- La carga isométrica del músculo ECU comportó una reducción $\operatorname{articular}^{23}$.

Por lo tanto, el efecto beneficioso del músculo ECU debe potenciarse durante el tratamiento conservador de las disfunciones LP en los pacientes con hiperlaxitud y sintomatología a nivel LP, y en las fases pre- y posoperatorias de las reconstrucciones del ligamento LP.

\section{Inestabilidad no disociativa carpiana palmar}

La CINDP se caracteriza por una flexión anormal de la hilera proximal, que pasa súbitamente a la extensión cuando la muñeca pasa de inclinación radial a cubital, lo cual genera un resalte que en ocasiones puede ser doloroso. Es una disfunción predominantemente cinemática y debería ser denominada discinesia de la hilera proximal para definir mejor el origen del problema. Por lo general, sucede en pacientes laxos ${ }^{4,24}$.

En una serie de especímenes se creó una CINDP seccionando los ligamentos que conectan la hilera proximal y la distal (piramidal-ganchoso y piramidal-hueso grande en la zona cubital, y ETT y escafo-capitate en la zona radial), y el ligamento radiocarpiano dorsal. Posteriormente, se valoró el efecto de la carga muscular isométrica aislada o combinada de los cinco músculos motores de la muñeca sobre la posición de la hilera proximal del carpo.

La carga isométrica aislada del músculo ECU fue la única que consiguió corregir la flexión de la primera hilera del carpo en posición neutra de la muñeca. Además, el efecto pronador de la hilera distal del carpo generada por este músculo impidió el resalte brusco en extensión de la hilera proximal durante la inclinación cubital de la muñeca ${ }^{25}$

Por lo tanto, el efecto beneficioso del músculo ECU en la estabilización de la hilera proximal del carpo en las muñecas con una CINDP debe potenciarse durante el tratamiento conservador de estas, sobre todo en pacientes hiperlaxos sintomáticos y en las fases pre- y posoperatoria de las reconstrucciones de los ligamentos mediocarpianos de la zona cubital. Por otro lado, las ortesis que generan una pronación de la hilera distal del carpo pueden ayudar a mejorar la sintomatología de las CINDP.

\section{Efecto de la alineación del radio distal sobre el control muscular}

En trabajos de laboratorio recientes, los autores de este artículo han reproducido diferentes tipos de malas uniones extraarticulares del radio distal al combinar angulaciones dorsales, volares y acortamientos. En cada una de ellas se ha valorado el efecto sobre el carpo de las cargas isométricas de los mismos músculos estudiados en los experimentos precedentes, en casos de ligamento EL competente e incompetente. Los resultados de los estudios demostraron que las malas alineaciones del radio alteran el comportamiento cinético del carpo de las siguientes maneras ${ }^{26}$ :

- Cuando la superficie articular del radio se halla en inclinación volar de más de $20^{\circ}$, el efecto de supinación intracarpiana que tenía la carga muscular simultánea de todos los músculos sobre el carpo se pierde. En esta situación, el efecto producido es justo el contrario: una pronación intracarpiana.

- Cuando la superficie articular del radio se halla en inclinación dorsal de más de $20^{\circ}$ y el carpo se somete a la carga de la musculatura, se genera una traslación dorsal del escafoides significativa (Figura 17).

- Si a la mala unión dorsal del radio se le añade un acortamiento de este de más de $4 \mathrm{~mm}$, la eficacia de los músculos supinadores para controlar y estabilizar el espacio articular EL se pierde. 
Figura 17. Espécimen fijado en el soporte en el que se ha creado una mala unión dorsal con acortamiento del radio distal y que se someterá a la simulación de la contracción muscular. A la derecha: esquema de lo que ocurre con el escafoides cuando este tipo de mala unión excede los $20^{\circ}$ de angulación dorsal y los $4 \mathrm{~mm}$ de acortamiento.

Fuente: Imágenes obtenidas durante la realización del estudio.
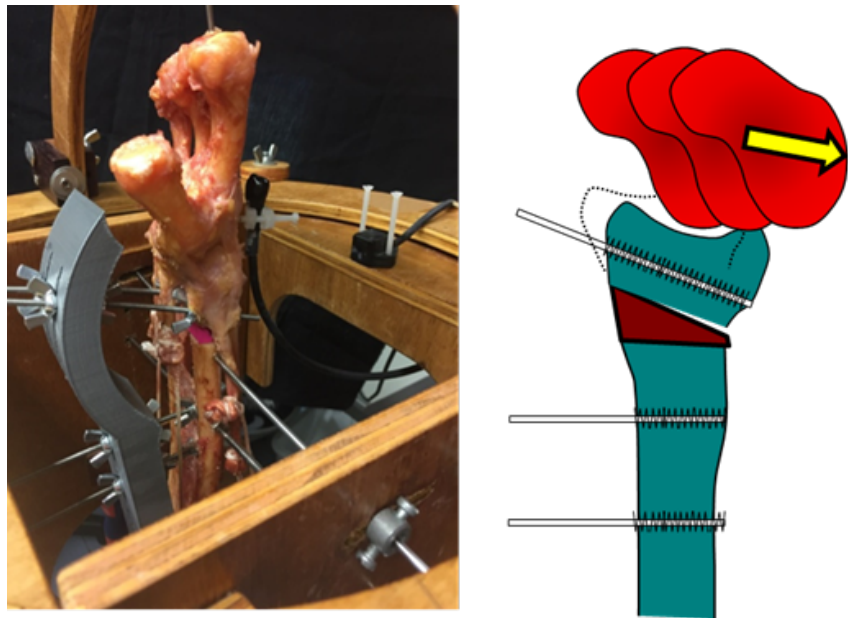

De estos estudios se deduce que ${ }^{26}$ :

- Cuando se asocia una fractura del radio distal y una lesión del ligamento EL, la alineación del radio en los planos coronal y sagital debe ser lo más próxima posible a la normalidad para poder realizar una estabilización dinámica muscular eficiente de la articulación EL.

- Una inestabilidad EL sintomática asociada a una mala unión del radio hacia dorsal y con acortamiento tratada solo con ligamentoplastia tiene altas posibilidades de fracasar.

- Una disfunción EL asociada a una mala alineación dorsal del radio tendrá, probablemente, una evolución más rápida hacia a una artropatía.

\section{El dolor cubital}

El estudio de Rein et al. ${ }^{27}$ sobre las estructuras ligamentosas y periarticulares de la zona cubital de la muñeca demostró que, a excepción del disco articular que forma la zona central del fibrocartílago triangular y el ligamento cúbito-semilunar, las partes blandas de la región cubital están ricamente inervadas. Estas estructuras contienen, en su mayoría, terminaciones nerviosas libres y corpúsculos nerviosos no clasificables que actúan como nociceptores y tienen un papel procioceptivo relevante ${ }^{27}$. Estos hallazgos ayudan a entender los siguientes aspectos sobre el dolor en la zona cubital de la muñeca:

- A diferencia del dolor en la zona radial, el dolor en la zona cubital suele provenir de lesiones de partes blandas ricamente inervadas.

- Esta es una región con gran importancia propioceptiva, donde la estabilización dinámica tiene un papel relevante.

- Las lesiones pequeñas, aunque no generen ninguna inestabilidad, pueden ser muy dolorosas.

- La reeducación propioceptiva es importante en el tratamiento conservador y en el pre- y posoperatorio de cirugías sobre las partes blandas de la zona.
- Se debe potenciar el uso de la artroscopia para mantener la propiocepción siempre que sea posible.

- Es preferible reparar las estructuras que todavía mantengan propiedades propioceptivas a usar injertos tendinosos.

\section{Conclusiones}

El trabajo en el laboratorio, además de permitir el aprendizaje y la investigación, ha generado algunas conclusiones no solamente científicas. En primer lugar, las ciencias básicas como la anatomía y la biomecánica son las claves para entender mejor cómo funciona la muñeca y, en particular, cómo aparecen las denominadas inestabilidades del carpo, pues su estabilidad no depende solo de uno o de varios ligamentos. En segundo, los fracasos terapéuticos o los resultados no satisfactorios deben despertar la curiosidad y es necesario ser críticos para poder analizarlos. Y en tercer lugar, el trabajo en equipo es la única manera de conseguir resultados.

\section{Conflicto de Interés}

Ninguno declarado por los autores.

\section{Financiación}

Ninguna declarada por los autores.

\section{Agradecimientos}

Ninguna declarada por los autores.

\section{Referencias}

1. Saffar P. Chondrocalcinosis of the wrist. J Hand Surg Br. 2004;29(5):486-93. DOI: 10.1016/j.jhsb.2004.02.013.

2. Romano S. Non-traumatic osteoarthritis of the wrist: chondrocalcinosis. Chir Main. 2003;22(6):285-92. DOI: 10.1016/j. main.2003.09.012.

3. Salva-Coll G, Garcia-Elias M, Lluch-Bergada A, Esplugas M, Llusa-Perez M. Kinetic dysfunction of the wrist with chronic scapholunate dissociation. A cadaver study. Clin Biomech (Bristol, Avon). 2020;77:105046. DOI: 10.1016/j.clinbiomech.2020.105046.

4. Garcia-Elias M. The non-dissociative clunking wrist: a personal view. J Hand Surg Eur Vol. 2008;33(6):698-711. DOI: 10.1177/1753193408090148.

5. Crisco JJ, Heard WM, Rich RR, Paller DJ, Wolfe SW. The mechanical axes of the wrist are oriented obliquely to anatomical axes. J Bone Joint Surg Am. 2011;93(2):169-77. DOI: 10.2106/ JBJS.I.01222.

6. Garcia-Elias M, Alomar X, Monill J. Dart-throwing motion in patients with scapholunate instability: a dynamic four-dimensional computed tomography study. J Hand Surg Eur Vol. 2014;39(4):346-52. DOI: 10.1177/1753193413484630.

7. Salva-Coll G, Garcia-Elias M, Leon-Lopez M, Llusa-Perez M, Rodriguez-Baeza A. Effects of forearm muscles on carpal stability. J Hand Surg Eur Vol. 2011;36(7):553-9. DOI: $10.1177 / 1753193411407671$. 
8. Esplugas M, Salva-Coll G, Lluch A, Garcia-Elias M. The scapholunate joint is reversely aligned when the wrist is axially loaded or axially tracted. A kinetic study in cadavers. Las Vegas: International Wrist Investigation Workshop; 2019.

9. Berger RA, Imeada T, Berglund L, An KN. Constraint and material properties of the subregions of the scapholunate interosseous ligament. J Hand Surg Am. 1999;24(5):953-62. DOI: 10.1053/jhsu.1999.0953.

10. Garcia-Elias M, Puig de la Bellacasa I, Schouten C. Carpal ligaments: a functional classification. Hand Clin. 2017;33(3):51120. DOI: 10.1016/j.hcl.2017.04.007.

11. Kamal R, Chehata A, Rainbow MJ, Llusá M, Garcia-Elias M. The effect of the dorsal intercarpal ligament on lunate extension after distal scaphoid excision. J Hand Surg Am. 2012;37(11):2240-5. DOI: 10.1016/j.jhsa.2012.07.029.

12. Esplugas M, Garcia-Elias M, Lluch-Bergada A, Fernández-Noguera N, Puig de la Bellacasa I. SL realignment using local tendon grafts: qualitative vector analysis of the two potential donors (FCR and ECRL). Copenhagen: Federation of European Societies for Surgery of the Hand; 2018.

13. Lluch A, Salvà G, Esplugas M, Llusá M, Hagert E, Garcia-Elias M. El papel de la propiocepción y el control neuromuscular en las inestabilidades del carpo. Rev Iberoam Cir Mano. 2015;43(1):70-8. DOI: 10.1016/j.ricma.2015.06.012.

14. Hagert E, Lluch A, Rein S. The role of proprioception and neuromuscular stability in carpal instabilities. J Hand Surg Eur Vol. 2016;41(1):94-101. DOI: 10.1177/1753193415590390.

15. Hagert E, Garcia-Elias M, Forsgren S, Ljung BO. Immunohistochemical analysis of wrist ligament innervation in relation to their structural composition. J Hand Surg Am. 2007;32(1):30-6. DOI: 10.1016/j.jhsa.2006.10.005.

16. Hagert E, Persson JKE, Werner M, Ljung BO. Evidence of wrist proprioceptive reflexes elicited after stimulation of the scapholunate interosseous ligament. J Hand Surg Am. 2009;34(4):64251. DOI: 10.1016/j.jhsa.2008.12.001.

17. Hagert E, Persson JK. Desensitizing the posterior interosseous nerve alters wrist proprioceptive reflexes. J Hand Surg Am. 2010;35(7):1059-66. DOI: 10.1016/j.jhsa.2010.03.031.
18. Hagert E, Ferreres A, Garcia-Elias M. Nerve-sparing dorsal and volar approaches to the radiocarpal joint. J Hand Surg Am. 2010;35(7):1070-4. DOI: 10.1016/j.jhsa.2010.03.032.

19. Salva-Coll G, Garcia-Elias M, Leon-Lopez MM, Llusa-Perez M, Rodriguez-Baeza A. Role of the extensor carpi ulnaris and its sheath on dynamic carpal stability. J Hand Surg Eur Vol. 2012;37(6):544-8. DOI: 10.1177/1753193411430921.

20. Esplugas M, Garcia-Elias M, Lluch A, Llusá M. Role of muscles in the stabilization of ligament-deficient wrists. J Hand Ther. 2016;29(2):166-74. DOI: 10.1016/j.jht.2016.03.009.

21. Holmes MK, Taylor S, Miller C, Brewster MBS. Early outcomes of "The Birmingham Wrist Instability Programme": a pragmatic intervention for stage one scapholunate instability. Hand Ther. 2017;22(3):90-100. DOI: 10.1177/1758998316685469.

22. Eraktas I, Ayhan C, Hayran M, Soylu A. Alterations in forearm muscle activation patterns after scapholunate interosseous ligament injury: a dynamic electromyography study. J Hand Ther. 2020;S0894-1130(20)30049-1. DOI: 10.1016/j.jht.2020.03.018.

23. León-Lopez MM, Salvà-Coll G, Garcia-Elias M, Lluch-Bergadà $\mathrm{A}$, Llusá-Pérez $\mathrm{M}$. Role of the extensor carpi ulnaris in the stabilization of the lunotriquetral joint. An experimental study. J Hand Ther. 2013;26(4):312-7. DOI: 10.1016/j.jht.2013.07.003.

24. Wolfe SW, Garcia-Elias M, Kitay A. Carpal instability nondissociative. J Am Acad Orthop Surg. 2012;20(9):575-85. DOI: 10.5435/JAAOS-20-09-575.

25. Lluch A, Esplugas M, Salvà G, Garcia-Elias M. The role of neuromuscular control in carpal non-dissociative instabilities. Seattle: American Society for Surgery of the Hand Annual Meeting; 2015.

26. Fernandez N, Garcia-Elias M, Esplugas M, Llusá-Pérez M, Lluch-Bergadá A, Puig de la Bellacasa I, et al. Influence of post traumatic deformities of the distal radius on muscle control of the carpus. A biomechanical study in cadaver. Federation of European Societies for Surgery of the Hand, FESSH - On(line) - Week; 2020.

27. Rein S, Semisch M, Garcia-Elias M, Lluch A, Zwipp H, Hagert E. Immunohistochemical mapping of sensory nerve endings in the human triangular fibrocartilage complex. Clin Orthop Relat Res. 2015;473(10):3245-53. DOI: 10.1007/ s11999-015-4357-z. 